Abstract

\title{
How Unique Is Wing-beating Sound? Classifying Mosquitoes from Wing-Beating Sounds ${ }^{\dagger}$
}

\author{
Xutong Wei ${ }^{1}$, Md Zakir Hossain ${ }^{2,3}$ and Khandaker Asif Ahmed ${ }^{4}$
}

Citation: Wei, X.; Hossain. M.Z.; Ahmed, K.A. How Unique Is Wingbeating Sound? Classifying Mosquitoes from Wing-Beating Sounds, in Proceedings of the 1st International Electronic Conference on Entomology, 1-15 July 2021, MDPI: Basel, Switzerland, doi:10.3390/IECE-10504

Published: 1 July 2021

Publisher's Note: MDPI stays neutral with regard to jurisdictional claims in published maps and institutional affiliations.

Copyright: (c) 2021 by the authors. Submitted for possible open access publication under the terms and conditions of the Creative Commons Attribution (CC BY) license (http://creativecommons.org/licenses /by/4.0/).

\author{
${ }^{1}$ Research School of Computing, The Australian National University (ANU), Canberra, ACT 2601, Australia \\ ${ }^{2}$ CSIRO Agriculture and Food, Black Mountain, Canberra, ACT 2601, Australia \\ ${ }^{3}$ Research School of Biology and Research School of Computing, ANU, Canberra, ACT 2601, Australia \\ ${ }^{4}$ CSIRO Land and Water, Black Mountain, Canberra, ACT 2601, Australia \\ + Presented at the 1st International Electronic Conference on Entomology (IECE 2021), 1-15 July 2021; \\ Available online: https://iece.sciforum.net/.
}

\begin{abstract}
Mosquitoes are the vectors of numerous deadly diseases including Yellow Fever, Malaria, Dengue fever, Zika and Chikungunya. Numerous control measures are being implemented around the world to suppress this notorious insect. Mosquito systematics is essential to study the mosquito prevalence in a definite area, which helps entomologists to design and implement specific control measures for prevalent species. Traditional systematics methods are laborious and time-consuming, which can be overcome by different image-processing based machine-learning (ML) approaches. But due to small size and poor-quality images, ML approaches often fail to identify mosquitoes from images. Recently, wing beating sounds showed potential to be used as a classifier in mosquito systematics. The current study aims to develop an ML model to identify different genera and species of mosquitoes, based on wing-beating sounds and propose a computationally inexpensive and fast tool to be used in mosquito control programmes. Wing beating sounds of six mosquito species of three different genera were collected from publicly available sources, and several state-of-art ML models were implemented on raw sounds as well as in processed spectrograms. On raw sounds, the general neural network model with 3 dense layers perceptrons reached at $59 \%$ accuracy, whereas for processed spectrograms, Resnet-18 enhance the accuracy to $86.1 \%$. The model also showed potential to enhance the accuracy for the identification of different mosquitoes' sexes. Our model has implications to be served as a mosquito prevalence monitoring tool and assist mosquito eradication program for specific mosquito genera, and finally will help medical practitioners to work on related pathogens.
\end{abstract}

Keywords: Mosquito; Machine Learning; Artificial Intelligence; Insect systematics; Wing-beating Sounds 